\title{
Sidewalk, a wayfinding message syntax for people with a visual impairment
}

\author{
Author(s) \\ van der Bie, Joey; Jaschinski, Christina ; Ben Allouch, Somaya \\ DOI \\ $10.1145 / 3308561.3354625$
}

Publication date

2019

Document Version

Author accepted manuscript (AAM)

Published in

ASSETS '19

\section{License}

Unspecified

Link to publication

\section{Citation for published version (APA):}

van der Bie, J., Jaschinski, C., \& Ben Allouch, S. (2019). Sidewalk, a wayfinding message syntax for people with a visual impairment. In J. P. Bigham, S. Azenkot, \& S. K. Kane (Eds.), ASSETS '19: the 21st International ACM SIGACCESS Conference on Computers and Accessibility (pp. 609-611). The Association for Computing Machinery. https://doi.org/10.1145/3308561.3354625

If you believe that digital publication of certain material infringes any of your rights or (privacy) interests,

please let the Library know, stating your reasons. In case of a legitimate complaint, the Library will make the material inaccessible and/or remove it from the website. Please contact the library:

https://www.amsterdamuas.com/library/contact/questions, or send a letter to: University Library (Library of the University of Amsterdam and Amsterdam University of Applied Sciences), Secretariat, Singel 425, 1012 WP Amsterdam, The Netherlands. You will be contacted as soon as possible. 


\section{Sidewalk, A Wayfinding Message Syntax for People with a Visual Impairment}

\author{
Joey van der Bie \\ Digital Life Centre \\ Amsterdam University of \\ Applied Sciences \\ Amsterdam, The Netherlands \\ j.h.f.van.der.bie@hva.nl
}

\author{
Christina Jaschinski \\ Saxion University of Applied \\ Sciences \\ Enschede, The Netherlands \\ c.jaschinski@saxion.nl
}

\author{
Somaya Ben Allouch \\ Digital Life Centre \\ Amsterdam University of \\ Applied Sciences \\ Amsterdam, The Netherlands \\ s.ben.allouch@hva.nl
}

\begin{abstract}
Traditional turn-by-turn navigation approaches often do not provide sufficiently detailed information to help people with a visual impairment (PVI) to successfully navigate through an urban environment. To provide PVI with clear and supportive navigation information we created Sidewalk, a new wayfinding message syntax for mobile applications. Sidewalk proposes a consistent structure for detailed wayfinding instructions, short instructions and alerts. We tested Sidewalk with six PVI in the urban center of Amsterdam, the Netherlands. Results show that our approach to wayfinding was positively valued by the participants.
\end{abstract}

\section{Author Keywords}

Assistive technology; Visually Impaired; Smartphone;

Wayfinding; Navigation; Natural Language; Spoken Messages

\section{CCS Concepts}

-Social and professional topics $\rightarrow$ Assistive technologies; -Human-centered computing $\rightarrow$ Natural language interfaces; Sound-based input / output; Smartphones; User studies; -Information systems $\rightarrow$ Geographic information systems; Location based services;

\section{INTRODUCTION}

Navigating an unknown route can be challenging, especially for people with a visual impairment (PVI). Traditional turnby-turn navigation applications are not optimized for PVI and often do not provide accurate and sufficiently detailed information for successful navigation [1]. A navigation app for PVI should support their orientation and navigation performance, and provide reassurance that the correct path is followed. To achieve this, an app should distinguish between different wayfinding needs (e.g. start/end, crossings, warnings/dangerous situations, orientation, progress, familiar or unfamiliar route) and provide the suitable type and amount of information $[5,6,13]$.

Permission to make digital or hard copies of part or all of this work for personal or classroom use is granted without fee provided that copies are not made or distributed for profit or commercial advantage and that copies bear this notice and the full citation on the first page. Copyrights for third-party components of this work must be honored For all other uses, contact the owner/author(s).

ASSETS '19, October 28-30, 2019, Pittsburgh, PA, USA

Copyright is held by the author/owner(s)

ACM ISBN 978-1-4503-6676-2/19/10.

http://dx.doi.org/10.1145/3308561.3354625

\section{THE SIDEWALK WAYFINDING SYNTAX EXPLAINED}

We present Sidewalk, a consistent but extendable wayfinding syntax that allows for attention, short and detailed messages. This is accomplished by defining optional and obligatory elements in the syntax. Obligatory elements convey action and destination information. Optional elements extend the short message syntax with more details, and are placed at a consistent location in the syntax. The syntax follows a logical flow: important attention messages are provided at the beginning of the syntax, while additional orientation information is provided after the actual navigation instruction (action and destination). This way the user does not have listen to the complete message to take action, thereby reducing the cognitive load. This syntax can be easily automated by a navigation app. Moreover, the user can create custom long or short message without much effort. Table 1 shows the Sidewalk syntax with an example instruction.

\section{Elements of the Sidewalk Syntax \\ Attention Indicator}

Attention indicators can be used to give special notice to an attention message: "Attention..; Warning...".

\section{Attention Message}

Provide attention messages when a route point that strongly influences the navigation pattern is within range (5 - 20 meters) $[5,6,14]$. When multiple items are close to each other, they are combined into one message: "Attention, you are approaching a parking garage entrance and exit, followed by poles".

\section{Current Orientation}

Current orientation messages should be short and provide identifiable information to confirm that the correct path is followed. This can be a confirmation of information from the previous navigation message, or a direct detectable object.

\section{Distance}

Provide absolute distance in meters when the action needs to be performed in more than 5 meters. Steps might be more comprehensible, but give a false sense of accuracy. Stride length differs per person and users will count their steps instead of focusing on the environment. Commit to units over 5 meters, outdoor navigation software does not have the accuracy of knowing the absolute distance between a user's location and the next route point. 


\section{Attention Indicator* - Attention Message* - Current Orientation* - Distance* - Action - Action Indicator* \\ - Destination - Orientation to Action \\ Example: "Warning, - obstruction on the sidewalk. - You are at the Wibautstraat with traffic at your right. - After 60 meters - turn left - at the trashcan - onto Mauritskade. - On your left you pass a bakery."}

Table 1. Sidewalk wayfinding syntax with example instruction *indicate optional sections.

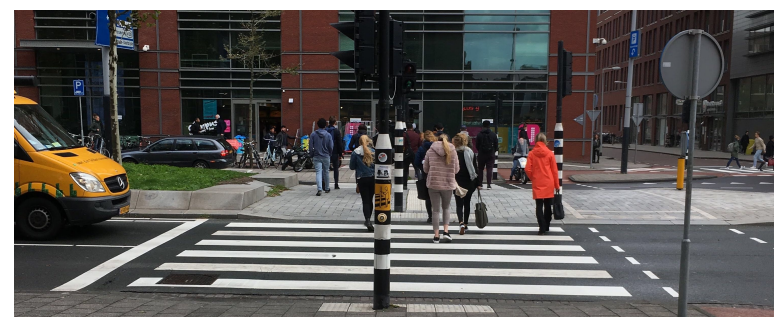

Figure 1. Example situation with Sidewalk instruction: "After 5 meters turn left at the tactile pavement and cross the road to Tweede Boerhaavestraat. You cross a bicycle lane, two roads with traffic lights and audio indicator, and a bicycle lane.

\section{Action}

Provide the action instruction accompanied with the direction in a short and simple format. Use physical directions (e.g. left, slight left). Only use more detailed direction indicators, such as clock positions, for finding a specific route point.

\section{Action Indicator}

Relative distances are preferred [3, 9, 10, 11, 14] but are not always possible. We propose to use both absolute and relative distance, so the user can get a better impression of the total distance and assess when an action needs to be performed. Provide relative distance indicators via route points or distinguishable objects where possible. Use objects that are hard to miss (avoiding the PVI searching for the object or becoming disorientated).

\section{Destination}

Provide the area name as is common for wayfinding messages. Although PVI often cannot detect street names [7], it is useful to become familiar with a route, functions as reference point in communication and for asking assistance [11].

\section{Orientation to Action}

Provide orientation information relevant for the user. Use the structure: "On your left..., on your right...".

\section{TESTING THE SIDEWALK WAYFINDING SYNTAX}

To test the wayfinding syntax a route in the urban center of Amsterdam was set out, including different route points that are perceived as challenging by PVI, e.g., obstacles [2, 3, 5]; crossroads [5, 7] and squares [5] (see Figure 1). The auditory wayfinding messages were provided by EyeBeacons [12], a wayfinding app designed for PVI. Bluetooth beacons were used to ensure precise triggering of the message in relation to the participant's location. Participants wore a bone-conducting headset and used their usual navigation aid (e.g., white cane) in addition to the provided instructions. To ensure the participant's safety, a researcher walked in close distance to the participant. In case of deviation participants were accompanied to the last route point and the instruction was repeated. After completing the route, participants were interviewed about their wayfinding experience. We used the System Usability Scale (SUS) (5-point Likert scale) [4] to measure overall experience of the Sidewalk syntax, the Raw NASA task-load index (11-point bipolar scale) (RTLX) [8] to measure mental an physical demand of the navigation performance, and open-ended questions to receive qualitative feedback.

\section{RESULTS}

Six PVI (two females, four males, age between 44 and 69 years) participated. Participants had different levels of visual acuity $(<2 \% \mathrm{n}=3,10-30 \% \mathrm{n}=1,>30 \% \mathrm{n}=2)$. All participants used a white cane. The SUS showed an average score of 84.2 $(\mathrm{SD}=10.4)$. All participants except one (p1) scored above the threshold of 68 points with values ranging from 82.5 (p3) to 95 points (p5). The RTLX scored also very positive, average load was low 17.3 out of 90 points. Qualitative feedback showed that all participants found the Sidewalk syntax to provide better information over traditional turn-by-turn navigation instructions, mostly due to the additional environment and orientation information. The Sidewalk syntax was found easy to understand and easy to use. Participants experienced the most difficulties at the two complex crossings and suggested to provide less information at these locations. All participants appreciated the different type of messages and suggested to implement this in existing navigation apps.

\section{CONCLUSION/ DISCUSSION}

We created Sidewalk, a new wayfinding message syntax to address the needs of PVI during wayfinding. Results show that our approach was positively perceived and most participants would like to use Sidewalk as their wayfinding approach. While our syntax can be considered a positive improvement over traditional turn-by-turn navigation, two participants (p1 and p3) were somewhat less positive than the other four participants. Both participants indicated to experience more mental and physical demand and p3 also felt more insecure during navigation. The researchers noticed that compared to the other participants, $\mathrm{p} 1$ and $\mathrm{p} 3$ were more insecure navigators in general. Therefore we suggest to take participants' general navigation confidence into account for future research. Furthermore, $\mathrm{p} 1$ found the messages too long and complex, and experienced difficulties with simultaneously listening to the messages and focusing on the environment As stated earlier, shortening and customization of the messages is possible using our syntax, thereby addressing the issues of message complexity. To further validate the usability of the syntax, in future research we would like to extend our test with more participants, different routes and apply to indoor routes.

\section{ACKNOWLEDGMENTS}

The authors would like to thank Corné Lukken, Michel Mercera and Geoffrey van Driessel, Royal Dutch Visio, Bartiméus, the HAN University of Applied Sciences and INFO. This work is supported by the ZonMW InZicht program, project nr. 94312006. 


\section{REFERENCES}

[1] Ali Abdolrahmani, Ravi Kuber, and Amy Hurst. 2016. An Empirical Investigation of the Situationally-induced Impairments Experienced by Blind Mobile Device Users. In Proceedings of the 13th Web for All Conference (W4A '16). ACM, New York, NY, USA, Article 21, 8 pages. DOI: http://dx.doi .org/10.1145/2899475.2899482

[2] Nikola Banovic, Rachel L. Franz, Khai N. Truong, Jennifer Mankoff, and Anind K. Dey. 2013. Uncovering Information Needs for Independent Spatial Learning for Users Who Are Visually Impaired. In Proceedings of the 15th International ACM SIGACCESS Conference on Computers and Accessibility (ASSETS '13). ACM, New York, NY, USA, Article 24, 8 pages. DOI :

http://dx.doi.org/10.1145/2513383.2513445

[3] Erin L. Brady, Daisuke Sato, Chengxiong Ruan, Hironobu Takagi, and Chieko Asakawa. 2015. Exploring Interface Design for Independent Navigation by People with Visual Impairments. In Proceedings of the 17th International ACM SIGACCESS Conference on Computers and Accessibility (ASSETS '15). ACM, New York, NY, USA, 387-388. DOI :

http://dx.doi.org/10.1145/2700648.2811383

[4] John Brooke. 1996. SUS: A "quick and dirty" usability scale. In P. W. Jordan, B. Thomas, B. A. Weerdmeester and A. L. McClelland (Eds.). Usability evaluation in industry 189, 194 (1996), 4-7.

[5] Florence Gaunet. 2006. Verbal guidance rules for a localized wayfinding aid intended for blind-pedestrians in urban areas. Universal Access in the Information Society 4, 4 (01 May 2006), 338-353. DOI :

http://dx.doi.org/10.1007/s10209-003-0086-2

[6] João Guerreiro, Dragan Ahmetovic, Kris M. Kitani, and Chieko Asakawa. 2017. Virtual Navigation for Blind People: Building Sequential Representations of the Real-World. In Proceedings of the 19th International ACM SIGACCESS Conference on Computers and Accessibility (ASSETS '17). ACM, New York, NY, USA, 280-289. DOI:

http://dx.doi.org/10.1145/3132525.3132545

[7] Richard Guy and Khai Truong. 2012. CrossingGuard: Exploring Information Content in Navigation Aids for Visually Impaired Pedestrians. In Proceedings of the SIGCHI Conference on Human Factors in Computing Systems (CHI '12). ACM, New York, NY, USA, 405-414. DOI :

http://dx.doi.org/10.1145/2207676.2207733
[8] Sandra G. Hart. 2006. Nasa-Task Load Index (NASA-TLX); 20 Years Later. Proceedings of the Human Factors and Ergonomics Society Annual Meeting 50, 9 (2006), 904-908. DOI: http://dx.doi.org/10.1177/154193120605000909

[9] Hugo Nicolau, Joaquim Jorge, and Tiago Guerreiro. 2009. Blobby: How to Guide a Blind Person. In CHI '09 Extended Abstracts on Human Factors in Computing Systems (CHI EA '09). ACM, New York, NY, USA, 3601-3606. DOI :

http://dx.doi.org/10.1145/1520340.1520541

[10] Daisuke Sato, Uran Oh, Kakuya Naito, Hironobu Takagi, Kris Kitani, and Chieko Asakawa. 2017. NavCog3: An Evaluation of a Smartphone-Based Blind Indoor Navigation Assistant with Semantic Features in a Large-Scale Environment. In Proceedings of the 19th International ACM SIGACCESS Conference on Computers and Accessibility (ASSETS '17). ACM, New York, NY, USA, 270-279. DOI :

http://dx.doi.org/10.1145/3132525. 3132535

[11] Morgan Klaus Scheuerman, William Easley, Ali Abdolrahmani, Amy Hurst, and Stacy Branham. 2017. Learning the Language: The Importance of Studying Written Directions in Designing Navigational Technologies for the Blind. In Proceedings of the 2017 CHI Conference Extended Abstracts on Human Factors in Computing Systems (CHI EA '17). ACM, New York, NY, USA, 2922-2928. DOI :

http://dx.doi.org/10.1145/3027063.3053260

[12] Joey van der Bie, Christina Jaschinski, and Somaya Ben Allouch. 2019. Communicating Multimodal Wayfinding Messages For Visually Impaired People Via Wearables. In Proceedings of the 21st International Conference on Human-Computer Interaction with Mobile Devices and Services: Adjunct (MobileHCI '19). ACM, Taipei, Taiwan. DOI :

http://dx.doi.org/10.1145/3338286.33444197

[13] Joey van der Bie, Britte Visser, Jordy Matsari, Mijnisha Singh, Timon van Hasselt, Jan Koopman, and Ben Kröse. 2016. Guiding the Visually Impaired Through the Environment with Beacons. In Proceedings of the 2016 ACM International Joint Conference on Pervasive and Ubiquitous Computing: Adjunct (UbiComp '16). ACM, New York, NY, USA, 385-388. DOI: http://dx.doi. org/10.1145/2968219.2971387

[14] Wayfindr. 2018. Wafindr 2.0 Open Standard. (14 March 2018). https://wwww.wayfindr.net/open-standard. 